\title{
Graphene-based infrared position-sensitive detector for precise measurements and high- speed trajectory tracking
}

Kaiyang Liu ${ }^{1, \dot{\dagger}}$, Wenhui Wang ${ }^{1, \dot{\dagger}}$, Yuanfang Yu ${ }^{1}$, Xiangyu Hou ${ }^{1}$, Yanpeng Liu ${ }^{2,3}$, Wei Chen ${ }^{2}$, Xiaomu Wang", Junpeng Lu*,1, Zhenhua Ni*,1

${ }^{1}$ School of Physics and Key Laboratory of MEMS of the Ministry of Education, Southeast University, Nanjing, 211189, China.

${ }^{2}$ Department of Chemistry and Centre for Advanced 2D Materials, National University of Singapore, 6 Science Drive 2, 117546, Singapore.

${ }^{3}$ Key Laboratory for Intelligent Nano Materials and Devices of Ministry of Education, and Institute of Nanoscience, Nanjing University of Aeronautics and Astronautics, Nanjing 210016, China

"National Laboratory of Solid State Microstructures, School of Physics, School of Electronic Science and Engineering, Collaborative Innovation Centre of Advanced Microstructures, Nanjing University, Nanjing, China.

${ }^{\dagger}$ These authors contributed equally to this work. 
*E-mail: zhni@seu.edu.cn (Z.N.) and phyljp@seu.edu.cn (J.L.). 


\section{Experimental Section:}

Fabrication of graphene-Ge PSDs. The N-doped Ge wafer with resistivity of 2-4 $\Omega \cdot \mathrm{cm}$ was used as substrate. A $40 \mathrm{~nm} \mathrm{SiO} 2$ insulating layer was deposited on $\mathrm{Ge}$ substrate, and the central region of $5 \mathrm{~mm} \times 5 \mathrm{~mm}$ area was removed by ultraviolet photolithography and HF etching. Atomic layer deposition was then employed to deposit a thin $\mathrm{Al}_{2} \mathrm{O}_{3}$ layer $(\sim 0.3 \mathrm{~nm})$ as recombination barrier (the thickness of $\mathrm{Al}_{2} \mathrm{O}_{3}$ layer is calculated and confirmed after 1000 circles ALD processes). Monolayer graphene was synthesized on copper foil catalyst surface in a low-pressure chemical vapor deposition system. The graphene-Ge PSD was fabricated by transferring graphene sheet onto the designed $\mathrm{Ge}$ substrate using wet transfer technique $\left(\mathrm{FeCl}_{3}\right.$ etching $)^{18}$. Two pairs of electrodes $(\mathrm{Ni}(5 \mathrm{~nm}) / \mathrm{Au}(50 \mathrm{~nm}))$ were deposited by using thermal evaporation with shadow mask. A thin layer of $\mathrm{Ni}(5 \mathrm{~nm}) / \mathrm{Au}(50 \mathrm{~nm})$ on the bottom of Ge acts as a common-grounded electrode.

Device Characterizations. The position-sensitive characteristics were carried out by moving the PSD fixed on the motorized stage. The photovoltage was recorded by a Keithley 2612 analyzer. In the wavelength-dependent experiment, lasers with various wavelengths were obtained by modulating the optical parametric oscillators of Coherent Chameleon pump. In the response speed measurement, an acoustic optical modulator was used to modulate the laser at a frequency of $10 \mathrm{kHz}$, and a digital storage oscilloscope was used to measure the transient response. In the experiment of trajectory tracking, a near IR LED (1300 nm, LED1300L from Thorlabs) was used as the light source, and the spin coater was used as the motion carrier. Two convex lenses with the 
focal length of 50 and $100 \mathrm{~mm}$ were placed between the light source and PSD to converge the light into the working area of the PSD. The signals from the four electrodes on the PSD were simultaneously measured by two cascaded 2612 analyzer, and used to locate the position of the target. The imaging software is pre-calibrated to eliminate the influence of the electrodes, e.g. asymmetrical contact resistance, the rectangular shape. All the experiments were carried out at room temperature in air. 


\section{Supplementary Details:}

The characteristics of the graphene film. To study the uniformity and integrality, the graphene film was transferred onto a $300 \mathrm{~nm} \mathrm{SiO} / / \mathrm{Si}$ substrate using wet-transfer technique. It can be seen from the optical image that graphene film is homogeneous and there are no winkles in a large area. Raman spectroscopy was carried out and no defect characteristic D peak was observed, which proves the high-quality of graphene.
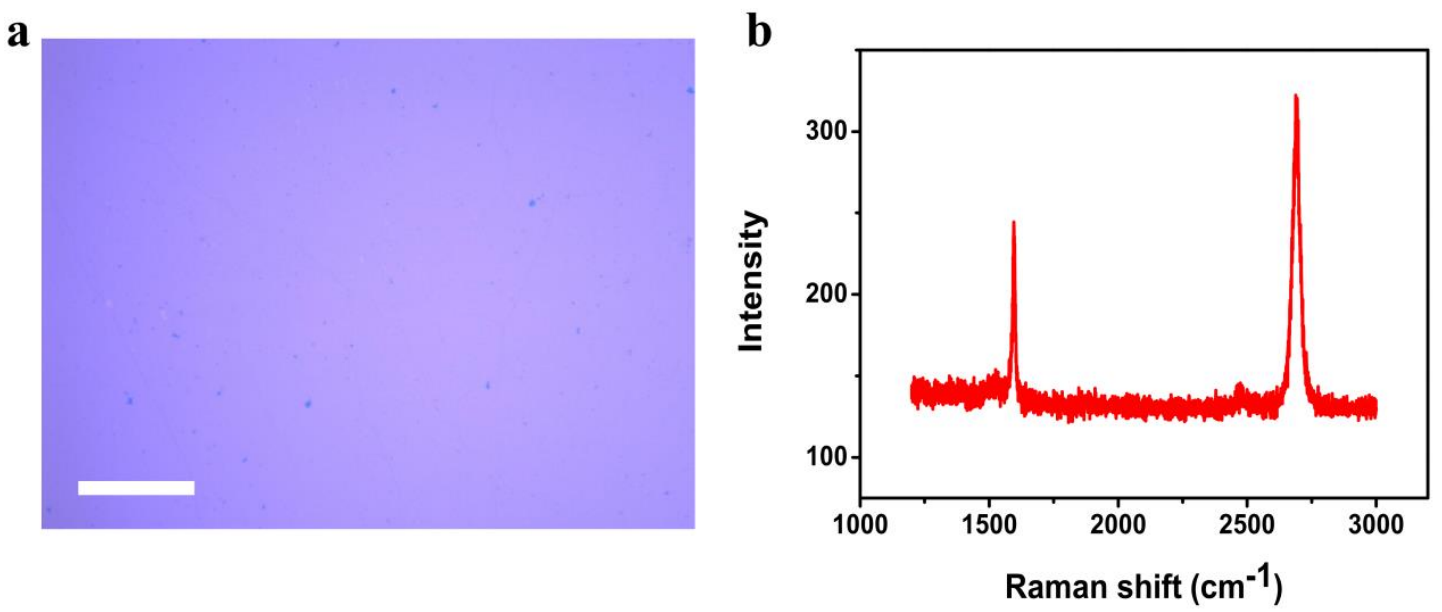

Figure S1. The characteristics of the graphene film. (a) Optical image of the graphene film on $300 \mathrm{~nm} \mathrm{SiO} / \mathrm{Si}$ substrate, scale bar is $100 \mu \mathrm{m}$. (b) Raman spectrum of monolayer graphene film.

The effect of $\mathrm{Al}_{2} \mathrm{O}_{3}$ layer. In addition to being directly collected by the electrode, the electrons in Ge can also recombine with holes in graphene during the diffusion process. This will reduce the number of holes reaching the electrode, which will degrade the output and the performance of PSD. In order to inhibit the recombination, ultrathin $\mathrm{Al}_{2} \mathrm{O}_{3}$ tunneling layer was inserted in between graphene and Ge. As shown in Figure S2a, the open circuit voltage of the heterojunction increases after the introduction of 
the tunneling layer, suggesting that the barrier height increases. The effect of tunneling layer thickness on the PSD is also studied. As shown in Figure S2b, the diffusion distance of holes in graphene increases with the increase of tunneling layer thickness in the first stage, which corresponds to the inhibition of recombination during the diffusion process. When the thickness is more than $\sim 0.3 \mathrm{~nm}$, the decrease of diffusion distance can be attributed to the decrease of separation efficiency of photo-induced carrier. The thick $\mathrm{Al}_{2} \mathrm{O}_{3}$ layer prevented the holes in $\mathrm{Ge}$ from entering the graphene at the illumination region, resulting in the decrease of the number of holes reaching the electrodes. Therefore, the optimized $\sim 0.3 \mathrm{~nm} \mathrm{Al}_{2} \mathrm{O}_{3}$ layer was selected as the tunneling layer for our PSD.
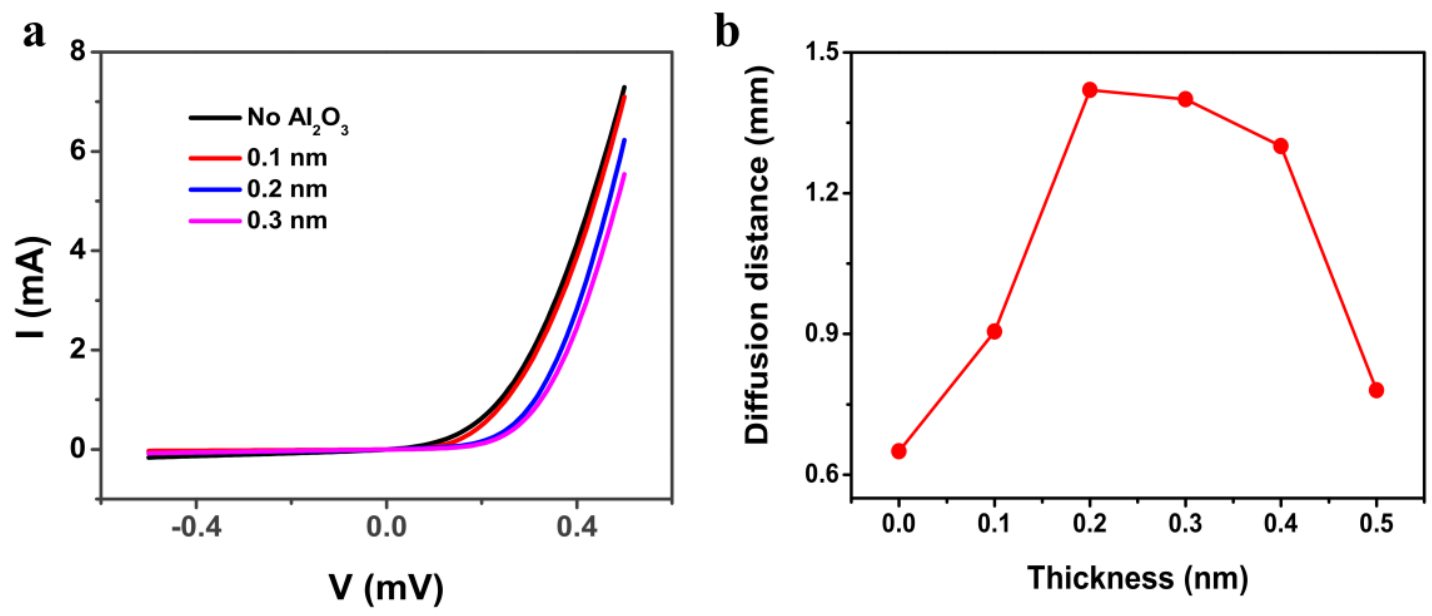

Figure S2. (a) The I-V curves of the graphene-Ge heterojunction with different thickness of $\mathrm{Al}_{2} \mathrm{O}_{3}$ layer on $\mathrm{Ge}$ substrate. (b) The relationship between carrier diffusing distance and $\mathrm{Al}_{2} \mathrm{O}_{3}$ thickness.

The effect of $\mathrm{SiO}_{2}$ layer. The electrons in Ge can also diffuse to the area beneath the electrodes and neutralize the holes collected from graphene, reducing the output photovoltage. In order to avoid this effect, $40 \mathrm{~nm} \mathrm{SiO} 2$ layer was introduced in between 
graphene and Ge substrate beneath the electrodes. As an insulating layer, it will prevent the electrons in Ge from being collected by the electrode. As shown in Figure S3, the output photo-voltage with $\mathrm{SiO}_{2}$ layer was significantly increased compared with that without $\mathrm{SiO}_{2}$ layer.

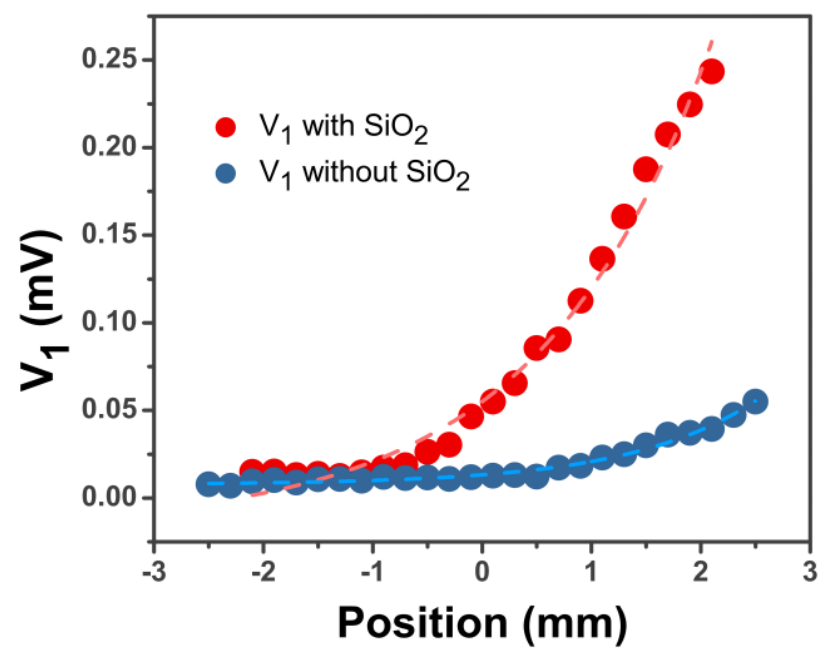

Figure S3. The output $\mathrm{V}_{1}$ with/without $40 \mathrm{~nm} \mathrm{SiO} 2$ layer in between graphene and $\mathrm{Ge}$ substrate, in the region beneath the electrodes.

The characteristics of graphene-Ge PSD. Nonlinearity, response speed and spatial resolution are important parameters to evaluate the performance of PSD. The nonlinearity $(\delta)$ characterizes the position detection error and is expressed as:

$$
\delta=\frac{2 \times \sqrt{\left[\sum_{i=1}^{N}\left(X_{i}-X_{i}^{T}\right)^{2}\right] / N}}{L} \times 100 \%
$$

where $X_{i}$ is the measured position, $X_{i}^{T}$ is the actual position, $N$ is number of measured position, and $L$ is the distance between the two electrodes. The nonlinearity of our PSD of $\sim 2.2 \%$ is extracted from Figure S4, which is much better than the 
industrial standard (15\%). Response speed is a key parameter that determines the application of PSD. Figure S5 shows the response time is dependent on the light position. It can be seen that response time increases with light spot away from the collecting electrode due to the increase of diffusion distance of carriers. Despite of the increase, our PSD can still realize high-frequency detection at hundreds of $\mathrm{KHz}$ level. Figure S6 displays the laser spot size independence photo-voltage and power dependence of spatial resolution. The PSD is built on lateral photoelectrical effect, therefore only the geometric center influence the photo-voltage. It can be seen that the spatial resolution is excellent under high power, and resolution degradation is inevitable with the decrease of incident power. The highest resolution of our PSD can reach $\sim 0.1$ $\mu \mathrm{m}$, which is comparable to or even better than commercial PSD. Our infrared PSD has large effective photosensitive area, works at room temperature, and is capable to realize rapid and highly sensitive position measurements, which laid a firm foundation for its further application in optical sensing systems.

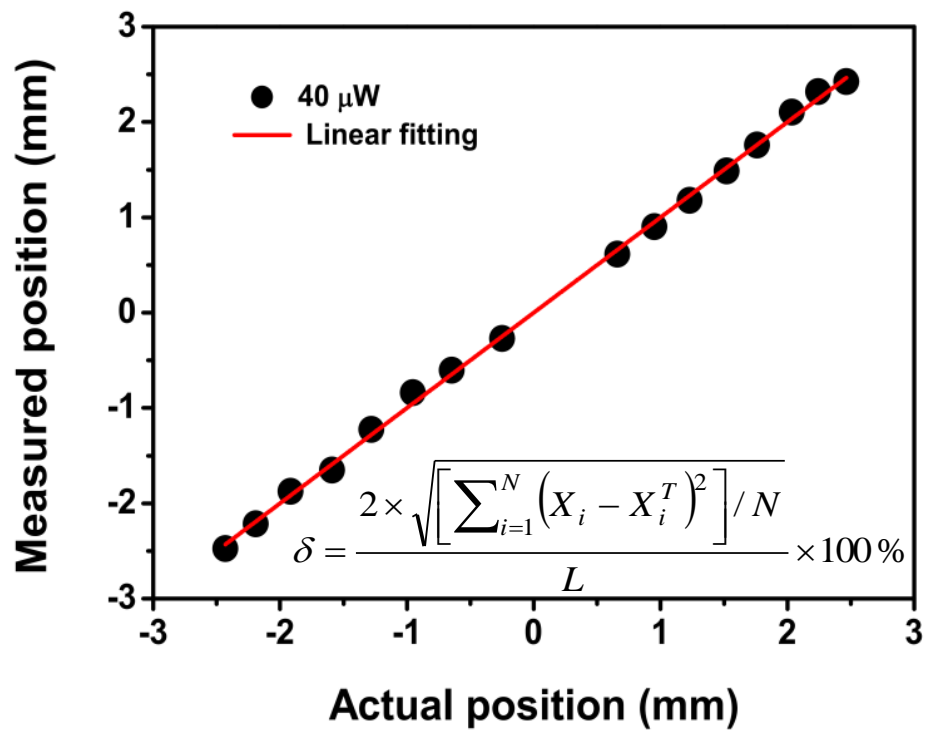


Figure S4. The position detection using graphene-Ge PSD, and the nonlinearity is calculated to be $\sim 2.2 \%$ by using the inset equation.

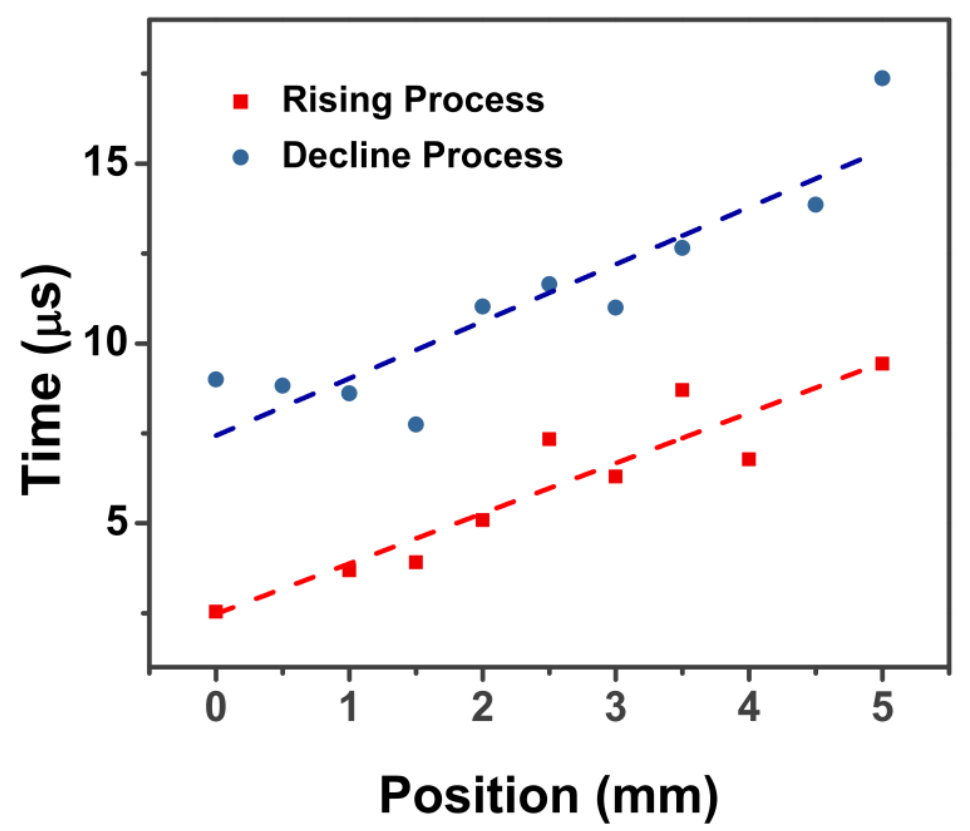

Figure S5. The response time of the PSD with the change of light position. 
$\mathbf{a}$

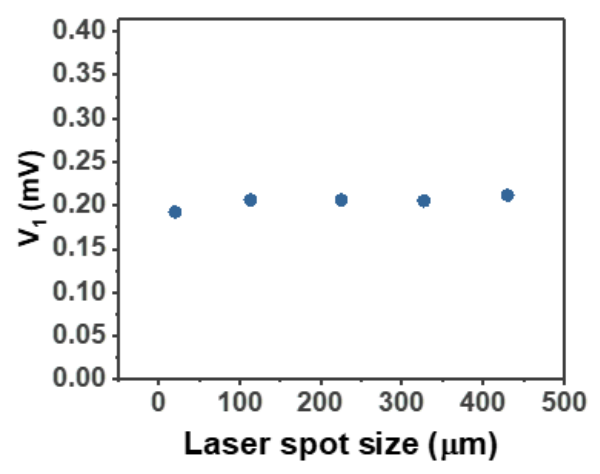

b

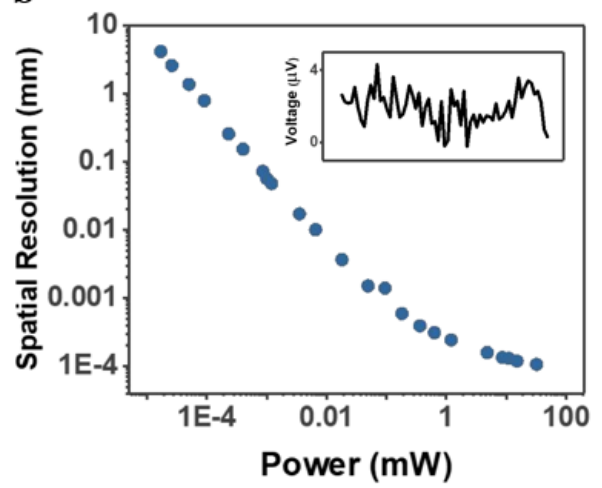

$\mathbf{C}$

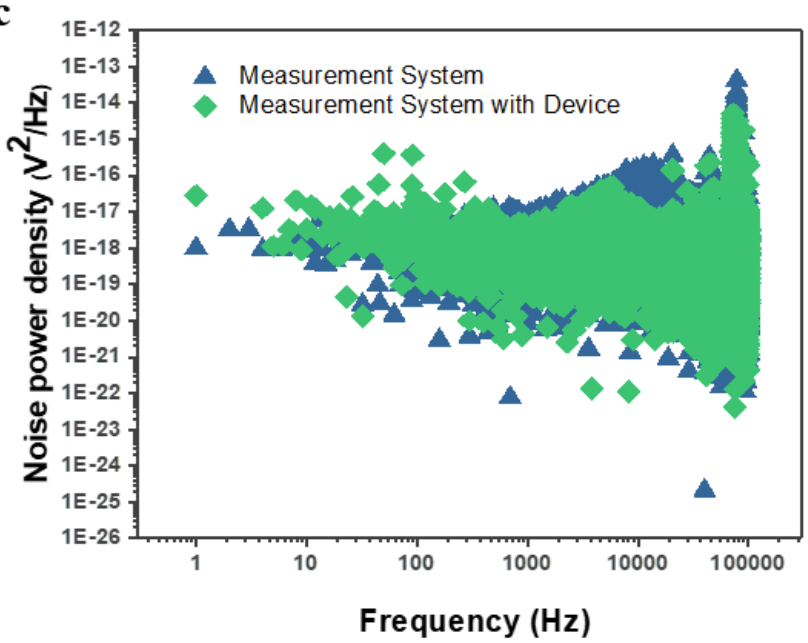

Figure S6. (a) The photo-voltage of the PSD is not influenced by laser spot size. (b) The spatial resolution of graphene-Ge PSD at different light power. Insert shows the dark voltage of the device. (c) The noise power density spectra of the instrument and device + instrument. The overall noise of the device and measurement system does not show significant difference to the noise of the individual measurement system.

Video S1. The demonstration of the non-contact optical sensing system for measuring sound frequencies. The left panel shows the system in real-time. The right-up panel shows a close-up of the graphene-Ge PSD, the laser light spot can be observed on the IR detector card. Meanwhile, when the IR detector card blocks the laser, the graphene- 
Ge PSD stops outputting signal to the speaker (VI) and volume of the music decrease. The right-bottom panel shows the setup diagram of the system. 